\title{
Comprehensive Analysis of Competing Endogenous RNA Network Focusing on Long Noncoding RNA Involved in Cirrhotic Hepatocellular Carcinoma
}

\author{
Yuli Zhang $\left(\mathbb{D},{ }^{1}\right.$ Dinggui Chen $\left(\mathbb{D},{ }^{2}\right.$ Miaomiao Yang $\left(\mathbb{D},{ }^{2}\right.$ Xianfeng Qian $\left(\mathbb{D},{ }^{2}\right.$ Chunmei Long $(\mathbb{D})^{2}$ \\ and Zhongwei Zheng ${ }^{2}{ }^{2}$ \\ ${ }^{1}$ Department of Pharmacy, The Third People's Hospital of Changzhou, Changzhou City, Jiangsu Province 213001, China
${ }^{2}$ Department of Gastroenterology, The Third People's Hospital of Changzhou, 300 Lanling North Road, Changzhou City,
Jiangsu 213001, China
}

Correspondence should be addressed to Zhongwei Zheng; zhengzw123@aliyun.com

Received 26 February 2021; Revised 9 May 2021; Accepted 4 June 2021; Published 23 June 2021

Academic Editor: Viswanathan Pragasam

Copyright (C) 2021 Yuli Zhang et al. This is an open access article distributed under the Creative Commons Attribution License, which permits unrestricted use, distribution, and reproduction in any medium, provided the original work is properly cited.

\begin{abstract}
The role of long noncoding RNAs- (lncRNAs-) associated competing endogenous RNA (ceRNA) in the field of hepatocellular carcinoma (HCC) biology is well established, but the involvement of lncRNAs competing interactions in the progression of liver cirrhosis to HCC is still unclear. We aimed to explore the differential expression profiles of lncRNAs, microRNAs (miRNA), and messenger RNAs (mRNAs) to construct a functional ceRNA network in cirrhotic HCC. The lncRNA, miRNA, and mRNA expression datasets were obtained from Gene Expression Omnibus and The Cancer Genome Atlas. Based on miRanda and TargetScan, the HCC-specific ceRNA network was constructed to illustrate the coexpression regulatory relationship of lncRNAs, miRNAs, and mRNAs. The potential prognostic indicators in the network were confirmed by survival analysis and validated by qRT-PCR. A total of 74 lncRNAs, 36 intersection miRNAs, and 949 mRNAs were differentially expressed in cirrhotic HCC samples compared with cirrhosis samples. We constructed a ceRNA network, including 47 lncRNAs, 35 miRNAs, and 168 mRNAs. Survival analysis demonstrated that 2 lncRNAs (EGOT and SERHL), 4 miRNAs, and 40 mRNAs were significantly associated with the overall survival of HCC patients. Two novel regulatory pathways, EGOT-miR-32-5p-XYLT2 axis and SERHL-miR-1269a/miR-193b-3p-BCL2L1/SYK/ARNT/CHST3/LPCAT1 axis, were built up and contribute to the underlying mechanism of HCC pathogenesis. The higher-expressed SERHL was associated with a higher risk of all-cause death. The expressions of SERHL-miR-1269a-BCL2L1 were significantly different using qRT-PCR in vitro studies. IncRNAs EGOT and SERHL might serve as effective prognostic biomarkers and potential therapeutic targets in cirrhotic HCC treatment.
\end{abstract}

\section{Introduction}

Liver cancer is the sixth most common incident carcinoma and the fourth most common cause of malignant tumor mortality [1], being estimated to be 18.1 million new patients and 9.6 million cancer-related deaths throughout the world in 2018 [2]. Accounting for over 85\% of primary liver cancers, hepatocellular carcinoma (HCC) is not evenly distributed among geographic regions, being accompanied by the increasing overall disease burden of HCC [3]. According to the etiology of cirrhosis, the risk factors for HCC mainly included hepatitis $B$ virus infection, hepatitis $C$ virus infection, alcohol, primary biliary cholangitis, autoimmune hepatitis, primary sclerosing cholangitis, and cryptogenic cirrhosis [4]. Therefore, liver cirrhosis plays a vital role in the pathogenesis of HCC. Based on the cirrhosis affecting progression to HCC, specific molecular biomarkers and potential regulatory mechanisms are essential and meaningful to the early diagnosis, treatment strategies, and the evaluation of prognosis.

Long noncoding RNAs (lncRNAs) are endogenous noncoding RNA molecules more than 200 nucleotides in length and can be subclassified into exonic, intronic, overlapping, and intergenic lncRNAs in nuclear or cytosolic fractions [5]. Given their regulation of cell proliferation, apoptosis, 
Volcano picture of GSE17967 diff IncRNA

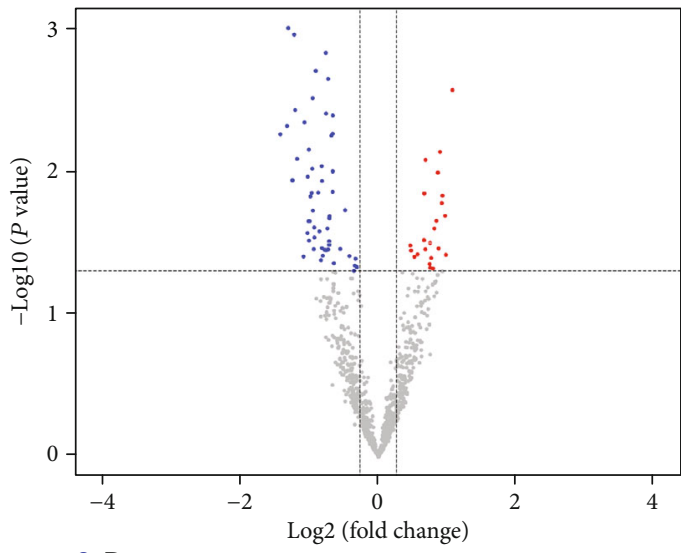

- Down

- Not

- Up

(a)

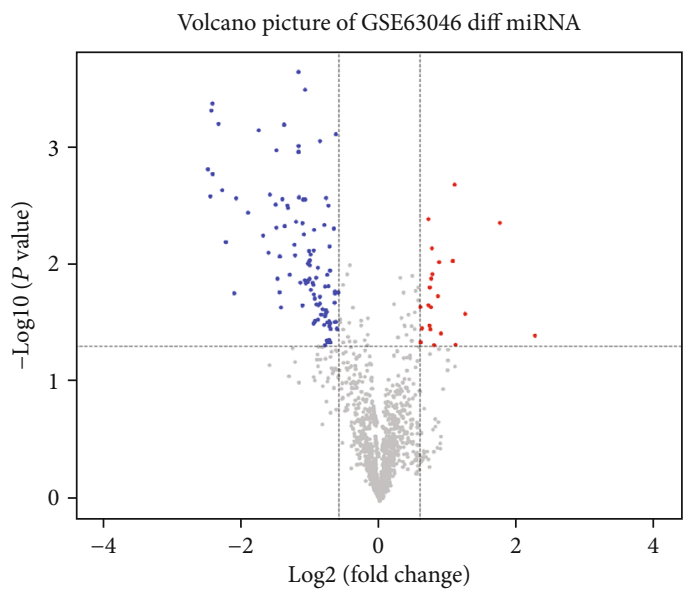

- Down

- Not

- Up
Volcano picture of GSE17967 diff mRNA

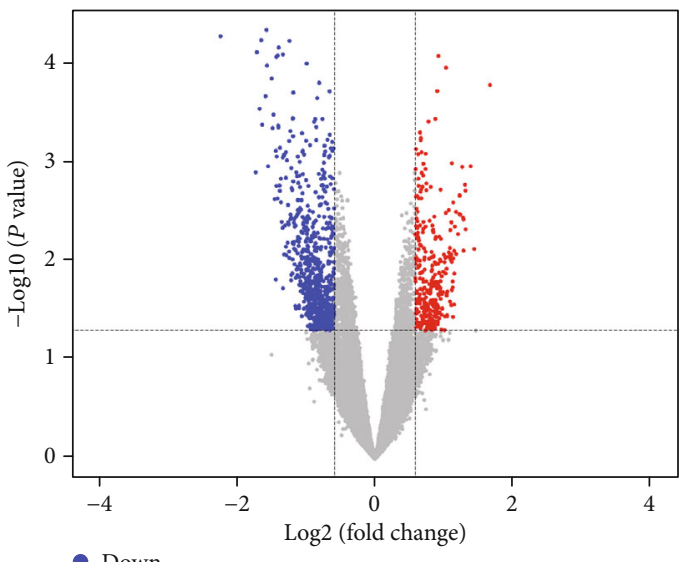

Down

Not

- Up

(b)

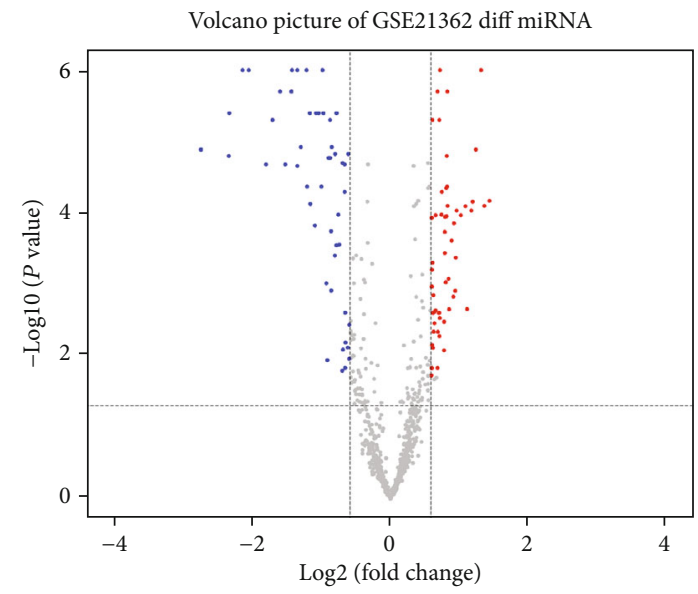

- Down

- Not

- Up

(c)

(d)

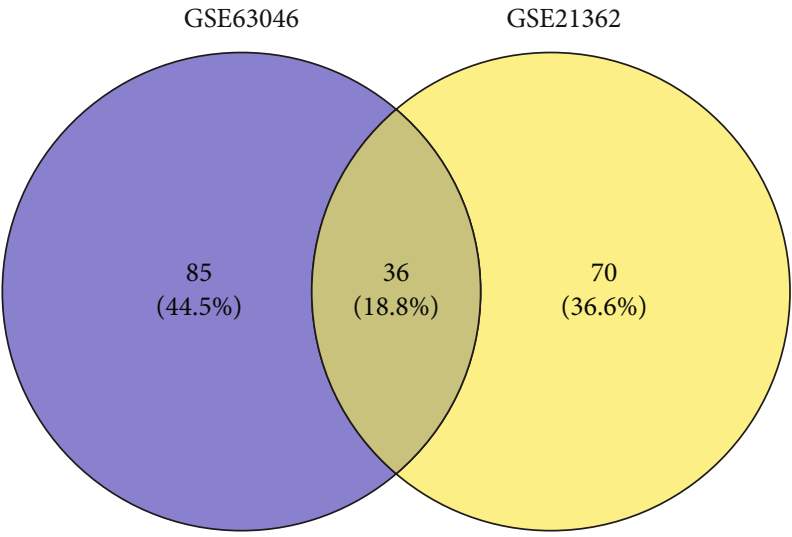

(e)

Figure 1: Differential analysis of lncRNAs, miRNAs, and mRNAs in cirrhotic patients with HCC compared with those in cirrhotic patients without HCC (fold change $>1.2 ; P<0.05)$. ( $\mathrm{a}, \mathrm{b})$ Volcano picture showing the upregulated (in red), downregulated (in blue), and undifferentiated (in gray) lncRNAs and mRNAs in their expression of GSE17967. (c, d) Volcano picture showing the upregulated (in red), downregulated (in blue), and undifferentiated (in gray) miRNAs in their expression of GSE63046 and GSE21362. (e) Venn diagram analysis of differentially expressed miRNA obtained from GSE63046 and GSE21362. The number in the overlapping portion indicates the common miRNAs. IncRNA: long noncoding RNA; miRNA: microRNA; mRNA: messenger RNA; HCC: hepatocellular carcinoma. 
TABLE 1: Differentially expressed lncRNAs between cirrhosis and cirrhotic HCC samples.

\begin{tabular}{|c|c|c|c|}
\hline $\operatorname{lncRNAs}$ & Regulation & Fold change & $P$ value \\
\hline BTF3P11 & Down & -2.663 & 0.006 \\
\hline NONHSAT060287.2 & Down & -2.494 & 0.005 \\
\hline XR_250850.2 & Down & -2.462 & 0.001 \\
\hline NR_027259.1 & Down & -2.361 & 0.012 \\
\hline NR_001284.2 & Down & -2.320 & 0.001 \\
\hline EGOT & Down & -2.299 & 0.004 \\
\hline NONHSAT039751.2 & Down & -2.256 & 0.008 \\
\hline NONHSAT126168.2 & Down & -2.113 & 0.040 \\
\hline NR_001554.2 & Down & -2.095 & 0.005 \\
\hline NONHSAT004151.2 & Down & -2.032 & 0.027 \\
\hline PMCHL1 & Down & -2.027 & 0.011 \\
\hline NR_001559.2 & Down & -2.010 & 0.023 \\
\hline ENST00000414544.1 & Down & -2.005 & 0.007 \\
\hline SERHL & Down & -2.002 & 0.031 \\
\hline ASMTL-AS1 & Down & -1.991 & 0.023 \\
\hline MAPKAPK5-AS1 & Down & -1.974 & 0.015 \\
\hline NONHSAT047922.2 & Down & -1.947 & 0.014 \\
\hline TMEM92-AS1 & Down & -1.937 & 0.010 \\
\hline NONHSAT121535.2 & Down & -1.929 & 0.003 \\
\hline NONHSAT145653.2 & Down & -1.925 & 0.019 \\
\hline NR_027434.2 & Down & -1.910 & 0.035 \\
\hline NONHSAT220650.1 & Down & -1.901 & 0.025 \\
\hline ENST00000562082.1 & Down & -1.898 & 0.029 \\
\hline XR_934561.1 & Down & -1.871 & 0.002 \\
\hline ENST00000420452.5 & Down & -1.827 & 0.014 \\
\hline PYY2 & Down & -1.805 & 0.027 \\
\hline NONHSAT157153.1 & Down & -1.775 & 0.042 \\
\hline NONHSAT041143.2 & Down & -1.762 & 0.009 \\
\hline NONHSAT220262.1 & Down & -1.759 & 0.035 \\
\hline ENST00000395400.4 & Down & -1.757 & 0.012 \\
\hline MLLT10P1 & Down & -1.745 & 0.039 \\
\hline ENST00000435356.2 & Down & -1.707 & 0.036 \\
\hline PKD1P6 & Down & -1.691 & 0.001 \\
\hline NONHSAT054874.2 & Down & -1.687 & 0.004 \\
\hline NONHSAT206981.1 & Down & -1.680 & 0.036 \\
\hline NONHSAT137305.2 & Down & -1.666 & 0.025 \\
\hline NACAP1 & Down & -1.655 & 0.002 \\
\hline LOC105376196 & Down & -1.637 & 0.031 \\
\hline ENST00000497872.3 & Down & -1.636 & 0.033 \\
\hline NONHSAT094826.2 & Down & -1.632 & 0.022 \\
\hline NONHSAT192084.1 & Down & -1.629 & 0.021 \\
\hline NONHSAT185078.1 & Down & -1.617 & 0.023 \\
\hline TNXA & Down & -1.587 & 0.005 \\
\hline NONHSAT002975.2 & Down & -1.581 & 0.014 \\
\hline NONHSAT119971.2 & Down & -1.561 & 0.044 \\
\hline NONHSAT212252.1 & Down & -1.465 & 0.035 \\
\hline NR_002174.2 & Down & -1.395 & 0.019 \\
\hline XR_918449.1 & Down & -1.337 & 0.039 \\
\hline
\end{tabular}

TABle 1: Continued.

\begin{tabular}{|c|c|c|c|}
\hline lncRNAs & Regulation & Fold change & $P$ value \\
\hline NONHSAT083938.2 & Down & -1.278 & 0.050 \\
\hline NR_135506.1 & Down & -1.268 & 0.046 \\
\hline NR_037932.1 & Down & -1.258 & 0.041 \\
\hline NONHSAT047228.2 & Down & -1.240 & 0.047 \\
\hline NONHSAT135135.2 & Up & 2.094 & 0.003 \\
\hline NONHSAT070712.2 & Up & 1.960 & 0.039 \\
\hline NONHSAT129571.2 & Up & 1.944 & 0.021 \\
\hline NONHSAT145215.2 & Up & 1.894 & 0.015 \\
\hline NONHSAT123438.2 & Up & 1.880 & 0.017 \\
\hline NONHSAT001301.2 & Up & 1.849 & 0.007 \\
\hline NONHSAT171169.1 & $\mathrm{Up}$ & 1.824 & 0.035 \\
\hline NONHSAT175076.1 & Up & 1.809 & 0.010 \\
\hline NONHSAT076682.2 & Up & 1.782 & 0.022 \\
\hline LOC105370914 & Up & 1.723 & 0.048 \\
\hline NONHSAT193357.1 & Up & 1.711 & 0.029 \\
\hline ENST00000621900.1 & Up & 1.693 & 0.041 \\
\hline NONHSAT010763.2 & Up & 1.677 & 0.048 \\
\hline NR_134476.1 & Up & 1.668 & 0.045 \\
\hline NONHSAT171432.1 & Up & 1.601 & 0.008 \\
\hline NR_026891.1 & $\mathrm{Up}$ & 1.598 & 0.035 \\
\hline NONHSAT023390.2 & $\mathrm{Up}$ & 1.579 & 0.014 \\
\hline NONHSAT165295.1 & $\mathrm{Up}$ & 1.575 & 0.031 \\
\hline NR_051972.1 & $\mathrm{Up}$ & 1.477 & 0.038 \\
\hline COX7A2P2 & $\mathrm{Up}$ & 1.430 & 0.040 \\
\hline NONHSAT130696.2 & Up & 1.384 & 0.036 \\
\hline NR_003945.1 & Up & 1.373 & 0.033 \\
\hline
\end{tabular}

lncRNA: long noncoding RNA; HCC: hepatocellular carcinoma.

autophagy, epithelial-mesenchymal transition, and angiogenesis, cancer-specific lncRNAs are involved in the initiation, aggravation, and metastasis of HCC [6] and can serve as detectable diagnostic markers and effective therapeutic targets for HCC [7]. In the competing endogenous RNA (ceRNA) hypothesis, IncRNAs could act as competitive binding sites for the target microRNA (miRNA) response elements and then regulate the expression of corresponding messenger RNAs (mRNAs) [8]. The HCC-related deregulated ceRNA network was built to reveal the candidate prognostic cytoplasmic lncRNAs by integrated analysis in 371 HCC tissues and 50 healthy tissues from The Cancer Genome Atlas (TCGA) database [9]. However, the comprehensive analysis of the IncRNA-miRNA-mRNA ceRNA regulatory network in the progression of liver cirrhosis to HCC remains poorly characterized and be worth being further researched.

Given this context, we compared the differential expression of lncRNAs, miRNAs, and mRNAs between cirrhosis tissues and cirrhotic HCC tissues in the National Center for Biotechnology Information Gene Expression Omnibus (GEO) database and constructed the IncRNA-miRNAmRNA ceRNA coexpression network. Finally, two lncRNAs were significantly correlated with the overall survival of 
TABLE 2: miRNAs targeting specific key lncRNAs in cirrhotic HCC.

\begin{tabular}{|c|c|}
\hline Key lncRNAs & miRNAs \\
\hline ASMTL-AS1 & hsa-miR-1268a, hsa-miR-193b-3p \\
\hline EGOT & hsa-miR-32-5p \\
\hline PKD1P6 & hsa-miR-1268a \\
\hline PMCHL1 & hsa-miR-1268a, hsa-miR-130b-3p \\
\hline PYY2 & hsa-miR-1268a \\
\hline SERHL & hsa-miR-1269a, hsa-miR-193b-3p \\
\hline XR_918449.1 & hsa-miR-130b-3p \\
\hline NR_134476.1 & $\begin{array}{l}\text { hsa-miR-139-5p, hsa-miR-150-5p, hsa-miR-199a-3p, hsa-miR-200a-3p, hsa-miR-214-5p, } \\
\text { hsa-miR-497-5p }\end{array}$ \\
\hline NR_051972.1 & $\begin{array}{l}\text { hsa-miR-125a-5p, hsa-miR-150-5p, hsa-miR-195-5p, hsa-miR-199a-5p, hsa-miR-214-3p, } \\
\text { hsa-miR-424-5p, hsa-miR-497-5p }\end{array}$ \\
\hline NR_037932.1 & hsa-miR-1269a \\
\hline NR_027434.2 & hsa-miR-1268a, hsa-miR-1269a, hsa-miR-193b-3p \\
\hline NR_026891.1 & $\begin{array}{l}\text { hsa-miR-10a-5p, hsa-miR-125a-5p, hsa-miR-139-5p, hsa-miR-145-3p, hsa-miR-150-5p, } \\
\text { hsa-miR-200a-3p, hsa-miR-200b-3p, hsa-miR-214-5p, hsa-miR-31-5p, hsa-miR-429, hsa-miR-542-3p }\end{array}$ \\
\hline NR_003945.1 & $\begin{array}{l}\text { hsa-miR-125a-5p, hsa-miR-130a-3p, hsa-miR-139-5p, hsa-miR-142-3p, hsa-miR-146a-5p, } \\
\text { hsa-miR-150-5p, hsa-miR-199a-3p, hsa-miR-214-3p, hsa-miR-214-5p, hsa-miR-223-3p, } \\
\text { hsa-miR-30a-3p, hsa-miR-31-5p, hsa-miR-338-3p, hsa-miR-542-3p }\end{array}$ \\
\hline NR_001554.2 & hsa-miR-193b-3p \\
\hline NONHSAT220650.1 & hsa-miR-1269a \\
\hline NONHSAT206981.1 & hsa-miR-1269a, hsa-miR-130b-3p, hsa-miR-193b-3p \\
\hline NONHSAT193357.1 & $\begin{array}{l}\text { hsa-miR-125a-5p, hsa-miR-132-5p, hsa-miR-139-3p, hsa-miR-146a-5p, hsa-miR-150-5p, } \\
\text { hsa-miR-181a-3p, hsa-miR-214-3p, hsa-miR-214-5p, hsa-miR-342-3p, hsa-miR-450a-5p }\end{array}$ \\
\hline NONHSAT175076.1 & hsa-miR-132-5p, hsa-miR-31-5p, hsa-miR-376c-3p, hsa-miR-424-5p \\
\hline NONHSAT171432.1 & hsa-miR-199a-5p, hsa-miR-214-5p, hsa-miR-31-5p, hsa-miR-342-3p \\
\hline NONHSAT171169.1 & $\begin{array}{l}\text { hsa-miR-139-3p, hsa-miR-150-5p, hsa-miR-214-3p, hsa-miR-214-5p, hsa-miR-223-3p, } \\
\text { hsa-miR-338-3p, hsa-miR-542-3p }\end{array}$ \\
\hline NONHSAT165295.1 & hsa-miR-145-3p, hsa-miR-199a-3p \\
\hline NONHSAT157153.1 & hsa-miR-1268a, hsa-miR-1269a \\
\hline NONHSAT145653.2 & hsa-miR-1268a, hsa-miR-1269a \\
\hline NONHSAT145215.2 & $\begin{array}{l}\text { hsa-miR-132-5p, hsa-miR-142-5p, hsa-miR-146a-5p, hsa-miR-30a-3p, hsa-miR-338-3p, } \\
\text { hsa-miR-424-5p, hsa-miR-497-5p }\end{array}$ \\
\hline NONHSAT137305.2 & hsa-miR-1268a, hsa-miR-1269a \\
\hline NONHSAT135135.2 & hsa-miR-150-5p, hsa-miR-214-3p, hsa-miR-497-5p \\
\hline NONHSAT130696.2 & hsa-miR-10a-5p \\
\hline NONHSAT129571.2 & $\begin{array}{l}\text { hsa-miR-10a-5p, hsa-miR-145-3p, hsa-miR-146a-5p, hsa-miR-199a-5p, hsa-miR-30a-3p, } \\
\text { hsa-miR-342-3p, hsa-miR-497-5p, hsa-miR-542-3p }\end{array}$ \\
\hline NONHSAT123438.2 & $\begin{array}{l}\text { hsa-miR-150-5p, hsa-miR-195-5p, hsa-miR-199a-3p, hsa-miR-214-3p, hsa-miR-338-3p, } \\
\text { hsa-miR-342-3p, hsa-miR-497-5p }\end{array}$ \\
\hline NONHSAT121535.2 & hsa-miR-1269a, hsa-miR-193b-3p \\
\hline NONHSAT119971.2 & hsa-miR-1269a, hsa-miR-130b-3p \\
\hline NONHSAT083938.2 & hsa-miR-1268a \\
\hline NONHSAT076682.2 & hsa-miR-132-5p, hsa-miR-200b-3p, hsa-miR-214-5p, hsa-miR-223-3p \\
\hline NONHSAT070712.2 & $\begin{array}{l}\text { hsa-miR-10a-5p, hsa-miR-130a-3p, hsa-miR-139-3p, hsa-miR-142-5p, hsa-miR-150-5p, } \\
\text { hsa-miR-195-5p, hsa-miR-214-5p, hsa-miR-223-3p, hsa-miR-30a-3p, hsa-miR-338-3p, } \\
\text { hsa-miR-424-5p, hsa-miR-497-5p }\end{array}$ \\
\hline NONHSAT060287.2 & hsa-miR-1268a, hsa-miR-193b-3p \\
\hline NONHSAT054874.2 & hsa-miR-1269a, hsa-miR-130b-3p \\
\hline NONHSAT047922.2 & hsa-miR-1269a \\
\hline NONHSAT039751.2 & hsa-miR-1268a, hsa-miR-130b-3p, hsa-miR-193b-3p \\
\hline
\end{tabular}


TABLe 2: Continued.

\begin{tabular}{lc}
\hline Key lncRNAs & miRNAs \\
\hline NONHSAT023390.2 & $\begin{array}{c}\text { hsa-miR-125a-5p, hsa-miR-130a-3p, hsa-miR-132-5p, hsa-miR-145-3p, hsa-miR-146a-5p, } \\
\text { hsa-miR-150-5p, hsa-miR-199a-5p, hsa-miR-200a-3p, hsa-miR-200b-3p, hsa-miR-429 } \\
\text { hsa-miR-125a-5p, hsa-miR-195-5p, hsa-miR-199a-5p, hsa-miR-200a-3p, hsa-miR-424-5p, } \\
\text { hsa-miR-497-5p } \\
\text { NONHSAT010763.2 } \\
\text { NONHSAT002975.2 } \\
\text { NONHSAT001301.2 } \\
\text { ENST00000621900.1 } \\
\text { ENST00000562082.1 } \\
\text { ENST00000435356.2 } \\
\text { ENST00000414544.1 } \\
\text { ENST00000395400.4 }\end{array} \quad \begin{array}{c}\text { hsa-miR-125a-5p, hsa-miR-150-5p, hsa-miR-214-3p, hsa-miR-338-3p } \\
\text { hsa-miR-1268a }\end{array}$ \\
\hline
\end{tabular}

miRNA: microRNA; lncRNA: long noncoding RNA; HCC: hepatocellular carcinoma.

cirrhotic HCC by the univariate Cox regression analysis, and their pair subnetworks were related to HCC-related signaling pathways. The previous correlational studies obtained the ceRNA expression in HCC samples and adjacent normal liver samples and explored the etiopathogenesis of normal liver to HCC. Hence, an integrated analysis of the ceRNA network to elaborate the biological processes and pathways on cancer-specific lncRNAs in cirrhotic HCC is still lacking. Our present study enrolled cirrhosis with and without HCC samples to focus on the pathologic process of cirrhosis to HCC, illuminating the underlying HCC pathogenesis of ceRNA coexpression network in patients with cirrhosis.

\section{Materials and Methods}

2.1. Microarray Datasets and Preprocessing. The lncRNAs, miRNAs, and mRNAs expression datasets were retrieved and downloaded from the GEO database by searching liver cirrhosis with and without HCC. GSE17967 (63 patients, 16 cirrhotic tissues with HCC, and 47 cirrhotic tissues without HCC, http://www.ncbi.nlm.nih.gov/geo/query/acc.cgi?acc= GSE17967) was included in our study and be used to analyze the differentially expressed lncRNAs and mRNAs [10]. Annotation pipeline of lncRNA transcripts was developed and obtained with Affymetrix Human Genome U133A 2.0 microarray. GSE21362 (73 patients, http://www.ncbi.nlm .nih.gov/geo/query/acc.cgi?acc=GSE21362) and GSE63046 (15 patients, http://www.ncbi.nlm.nih.gov/geo/query/acc .cgi?acc=GSE63046) were enrolled to profile and overlap the differentially expressed intersection miRNAs [11, 12]. The characteristics of each dataset were shown in Table S1. The expressions of specific lncRNAs, miRNAs, and mRNAs in cirrhotic HCC patients were downloaded from the National Institutes of Health TCGA Data Portal. TCGALIHC (377 patients, https://portal.gdc.cancer.gov/projects/ TCGA-LIHC) was obtained for survival analysis. Differentially expressed lncRNAs, miRNAs, and mRNAs were extracted with restricted criteria, which were set at fold change $(\mathrm{FC})>1.2$ and $P<0.05$. Figure $\mathrm{S} 1$ depicts the flowchart for bioinformatics analysis, pointing out a cluster of lncRNA, miRNA, and mRNA.
2.2. Construction of the ceRNA Network. The regulatory network of lncRNAs, miRNAs, and mRNAs was constructed to demonstrate the regulatory relation. The ceRNA hypothesis is that lncRNAs, acting as the specific miRNA sponges, can bind to target miRNAs and then regulate corresponding mRNAs expression. We performed the lncRNAs-mRNAs network to construct the coexpression regulatory relation by estimating across the array (Fig. S2). The miRanda (http://www.microrna.org/) and TargetScan (http://www .targetscan.org/) were performed to predict the target miRNA, which play a negative role in regulating the expression of lncRNA and mRNA. Next, we overlapped the intersection datasets both in miRanda and TargetScan. Finally, we combined the IncRNA-miRNA and miRNA-mRNA pairs to build the ceRNA network, which was visualized by using Cytoscape v2.8.2 [13].

2.3. Survival Analysis. To investigate the prognostic significance of differentially expressed IncRNAs, miRNAs, and mRNAs, we performed the survival analysis by collecting the ceRNA expression and clinical information in 377 cirrhotic HCC samples from TCGA-LIHC database. We used the survival data to evaluate the association between overall survival in cirrhotic HCC and candidate corresponding RNA expression, which split into high-expression and low-expression groups using the median. Kaplan-Meier survival analysis with log-rank test was applied and compared to estimate the significant difference by a cutoff of $P<0.05$. The statistically significant lncRNAs in the survival analysis were used to reconstruct a new ceRNA network by Cytoscape v2.8.2. Baseline characteristics for IncRNAs EGOT and SERHL were depicted in Table S2. To clarify the associations with overall survival and lncRNAs EGOT and SERHL, we analyzed the clinical characteristics of enrolled patients, including age, sex, race, pathologic stage, Child-Pugh score, neoplasm histologic grade, Ishak fibrosis score, vascular invasion, residual tumor grade, radiation therapy, neoadjuvant therapy, embolization therapy, family history of cancer, risk factors, and biomarkers (AFP and albumin) (Table S3).

2.4. Cell Culture and Quantitative Real-Time Polymerase Chain Reaction ( $q$ RT-PCR). We obtained the Huh7 human 
TABLE 3: miRNAs targeting specific mRNAs in cirrhotic HCC.

\begin{tabular}{|c|c|}
\hline miRNAs & mRNAs \\
\hline hsa-miR-497-5p & $\begin{array}{l}\text { ARHGEF12, ASPH, B4GALT1, BTN1A1, CCND1, CD84, CDCA4, EED, EYA4, GINS4, } \\
\text { MTAP, NUP50, OTUB2, OTUD4, SEMA6D, SLC45A2, SPEN, USP12 }\end{array}$ \\
\hline hsa-miR-1268a & BMP1, CCNF, EHD2, IP6K1, LDB3, PAX8, SCGB1A1, SIGLEC8, TGFB1I1 \\
\hline hsa-miR-200a-3p & $\begin{array}{l}\text { AAK1, AQP4,DTL, FRK, G3BP1, KLF12, MARCH6, MPZL2, PANK3, RORA, SLC35A3, } \\
\text { ST3GAL6, STRN, TAPT1, THAP9, USP53, WDR59, WNK1, ZEB1, ZMYM6 }\end{array}$ \\
\hline hsa-miR-150-5p & $\begin{array}{l}\text { ACSL6, ADH6, B4GALT1, CD84, DIO2, MARCH6, NTRK2, PRKAA2, RAD23B, } \\
\text { RCAN1, SLC4A4, ZEB1 }\end{array}$ \\
\hline hsa-miR-338-3p & $\begin{array}{l}\text { AAK1, ACVR2B, CLN8, DGCR2, DTNA, FRK, GABRA4, GP2, MARCH6, NTRK2, } \\
\text { PRKAA2, RNASEL, SEMA6D, SSX2IP, ZBTB43, ZG16 }\end{array}$ \\
\hline hsa-miR-30a-3p & $\begin{array}{l}\text { AQP4, ASPH, CCDC186, CDK6, DDX17, DTL, ERBB4, FBXO4, FLRT3, HACD3, MOB1A, } \\
\text { MTAP, NTRK2, PRKAA2, PRRC1, STRN, TAP2, TMEM106B, VCPIP1 }\end{array}$ \\
\hline hsa-miR-146a-5p & $\begin{array}{l}\text { AAK1, CD84, DTNA, ERBB4, ETV6, FRK, KLF12, MTAP, MYO1B, NTRK2, PRKAA2, } \\
\text { RNASEL, SCD, SIKE1, SORT1, SSX2IP, UBE2W }\end{array}$ \\
\hline hsa-miR-1269a & BCL2L1, FAM120C, FOXE1, MXD3, RGS4, SYK \\
\hline hsa-miR-214-3p & $\begin{array}{l}\text { ACVR2B, BAG5, CD84, CLN8, CRB1, DGCR2, EHHADH, FRK, KCNJ9, KRAS, LHX6, } \\
\text { NEU3, SEMA6D, SLC2A5, TMEM106B }\end{array}$ \\
\hline hsa-miR-424-5p & $\begin{array}{l}\text { ASPH, B4GALT1, BTN1A1, CD84, CDCA4, EED, EYA4, GINS4, MTAP, NUP50, OTUB2, } \\
\text { OTUD4, SEMA6D, SLC45A2, SLC4A4, SPEN }\end{array}$ \\
\hline hsa-miR-214-5p & ABHD2, CCND1, CD84, DGCR2, GABBR2, KLF12, SCD, SLC28A3, ZKSCAN5 \\
\hline hsa-miR-193b-3p & ADGRE5, ARNT, BAZ2A, CHST3, COMMD9, LPCAT1, MPPED2, NPC1L1, RPS21 \\
\hline hsa-miR-31-5p & $\begin{array}{l}\text { AK4, AP3D1, BTN1A1, DYRK1A, EIF5A2, EPB41L5, FAM169A, FRK, G3BP1, HLF, } \\
\text { MAP3K1, NXPH3, SEMA6D }\end{array}$ \\
\hline hsa-miR-199a-5p & $\begin{array}{l}\text { ACVR2B, ARHGEF12, DYRK1A, ETV6, FLRT3, MARCH6, NEU3, NPY2R, } \\
\text { SLC35A3, SMC4, SOS2, ST3GAL6 }\end{array}$ \\
\hline hsa-miR-195-5p & $\begin{array}{l}\text { AK4, ARHGEF12, B4GALT1, EED, EYA4, MTAP, NUP50, OTUB2, OTUD4, } \\
\text { SEMA6D, SLC4A4, SPEN }\end{array}$ \\
\hline hsa-miR-125a-5p & AAK1, ASPH, ENPP1, FRK, PRKAA2, RORA, SIRT5, SLC2A5, SMG1 \\
\hline hsa-miR-139-5p & ASPH, ATRX, CD164, CDCA4, MARCH6, PSME4, SSX2IP, TMPO, TNFRSF11A, ZEB1 \\
\hline hsa-miR-223-3p & BCLAF1, CASP10, CENPN, KCNJ9, NUCKS1, PIK3C3, SLC4A4, TAP2, VCPIP1 \\
\hline hsa-miR-200b-3p & DTNA, MRPL19, MTAP, NRIP1, OXR1, PIK3C3, SORT1, UTY, ZEB1, ZNF674 \\
\hline hsa-miR-10a-5p & AAK1, ARHGEF12, CCDC177, CCDC186, CCDC88A, ELOVL2, RORA, SORT1, TGFBR1 \\
\hline hsa-miR-342-3p & AK4, AQP4, CASP2, CDK6, CLN8, G3BP1, SLC16A1, SLC45A2, ZKSCAN5 \\
\hline hsa-miR-130a-3p & AAK1, ALDH3A2, DEPDC1, EPB41L5, NEU3, PRKAA2, VCPIP1, WNK1 \\
\hline hsa-miR-429 & CCDC177, DCAF17, DTNA, EIF5A2, MRPL19, NTRK2, OXR1, UBE2W, ZEB1 \\
\hline hsa-miR-132-5p & CCND1, CRAT, DIO2, GABRD, SCD, ZG16 \\
\hline hsa-miR-199a-3p & ACVR2B, DIO2, EPB41L5, ERBB4, NTRK2, TMEM106B \\
\hline hsa-miR-130b-3p & ADCY1, KCNN3, SHANK2, ST18 \\
\hline hsa-miR-542-3p & B4GALT1, CDK6, DBT, EDEM3, UFL1 \\
\hline hsa-miR-142-5p & CDK6, ERBB4, IL6ST, OTUD4, PIK3C3, SIKE1 \\
\hline hsa-miR-145-3p & $\mathrm{CD} 84$ \\
\hline hsa-miR-376c-3p & CCDC186, DIO2, KRAS, PANK3, SMAD5 \\
\hline hsa-miR-142-3p & CD84, PSME4, SMG1, TGFBR1 \\
\hline hsa-miR-139-3p & ABHD2, TAP2 \\
\hline hsa-miR-32-5p & AFF3, ARHGEF17, GID4, XYLT2 \\
\hline hsa-miR-181a-3p & CPT1A, SLCO1B3, ZNF3 \\
\hline hsa-miR-450a-5p & PHACTR1, SMG1 \\
\hline
\end{tabular}

miRNA: microRNA; mRNA: messenger RNA; HCC: hepatocellular carcinoma.

hepatoma cell line and LX-2 human hepatic fibrosis cell line from the Shanghai Institute of Biochemistry and Cell Biology (Shanghai, China). Huh7 cells were cultured with the Dulbecco's Modified Eagle's Medium (Thermo Fisher Scien- tific, Waltham, MA) containing $10 \%$ fetal bovine serum (FBS), penicillin $(100 \mathrm{U} / \mathrm{mL})$, L-glutamine $(2 \mathrm{mM})$, and streptomycin $(100 \mu \mathrm{g} / \mathrm{mL})$ at $37^{\circ} \mathrm{C}$ in a humidified incubator $\left(5 \% \mathrm{CO}_{2}\right)$. LX-2 cells were cultured in Medium 199 (Sigma- 


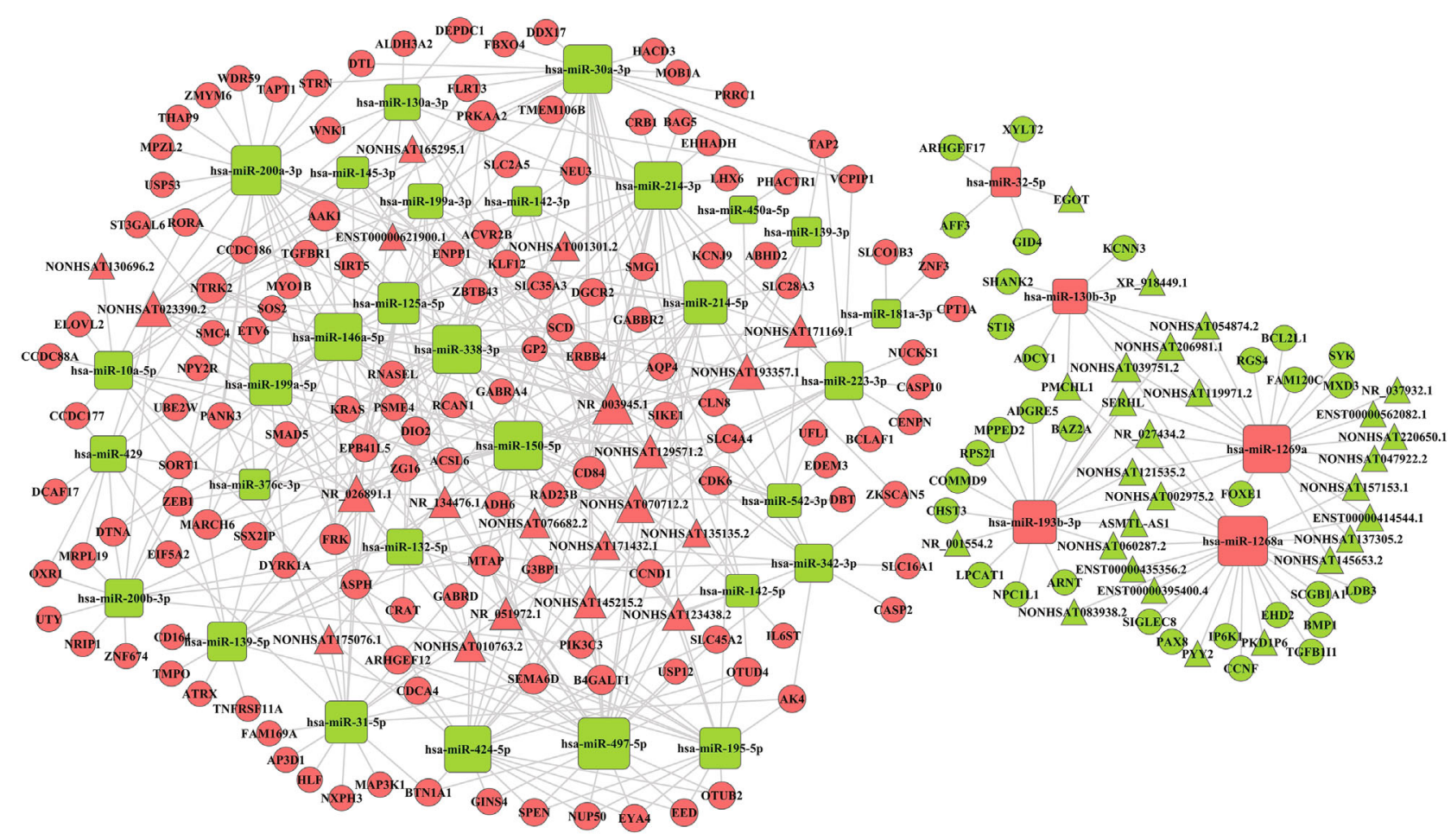

FIGURE 2: The ceRNA network of lncRNA-miRNA-mRNA. Triangles represent lncRNAs; rectangles with rounded corners represent miRNA; balls represent mRNAs. Red represents upregulated RNAs, and green represents downregulated RNAs. lncRNA: long noncoding RNA; miRNA: microRNA; mRNA: messenger RNA.

Aldrich, St. Louis, Missouri, USA) containing $10 \%$ FBS in a $5 \% \mathrm{CO} 2$ atmosphere at $37^{\circ} \mathrm{C}$. All the cell lines were seeded in microplates and maintained for 14 days. Total RNA was collected by a miRNeasy Mini Kit (Qiagen, Hilden, Germany) under the manufacturer's instructions. Then, the quantity, integrity, and purity were assessed using a NanoDrop 2000 (Thermo Fisher Scientific, MA, USA). $50 \mathrm{ng}$ of RNA was reverse-transcribed to cDNA by an iScript cDNA Synthesis Kit (170-8891, Bio-RAD). Quantitative PCR was performed using the 7900HT Rapid Real-Time Biosystems using SYBR-GREEN (170-8882AP, BIO-RAD) technology. Primers are listed in Table S4.

2.5. Statistical Analyses. Categorical variables were expressed as frequencies and proportions and compared using chisquare, Pearson's chi-squared, or Fisher exact test. Continuous variables were expressed as means with standard deviation (SD) or medians with interquartile ranges (IQRs) and compared using Student's $t$-tests or Wilcoxon rank-sum test. The absolute values of fold change $>1.2$ and $P<0.05$ were considered to indicate significant difference. The univariate and multivariate Cox proportional hazard regression models were conducted for discovering the connections between ceRNAs and overall survival. Backward stepwise selection was applied by using Akaike's information criterion to identify variables for multivariable Cox proportional hazard regression analysis. Then, we constructed a nomogram based on the multivariable Cox regression analyses to visualize the probability of 5-year survival in HCC patients. Variance inflation factor (VIF) assessed the multicollinearity of inde- pendent variables in the Cox regression model (VIF $\geq 10$ suggests multicollinearity). Hazard ratios (HRs) were presented with their 95\% confidence intervals (CIs). All analyses were implemented using the SPSS software (version 21.0, IBM, Chicago, Illinois, USA) and the R software (version 3.6.2, $\mathrm{R}$ Development Core Team, Vienna, Austria).

\section{Results}

3.1. Differential Expression of $\operatorname{lnc} R N A s$, miRNAs, and $m R N A s$ in Cirrhotic HCC. With the tumorigenesis of HCC in cirrhotic patients, a total of 74 lncRNAs from GSE17967, 36 intersection miRNAs from GSE21362 and GSE63046, and 949 mRNAs from GSE17967 were identified with the restricted criteria of FC $>1.2$ and $P<0.05$ by bioinformatics analysis (Figure 1). The 74 HCC-specific differentially expressed lncRNAs were composed of 22 upregulated and 52 downregulated lncRNAs in GSE17967 (Figure 1(a); Table 1; Table S5). For 949 differentially expressed mRNAs in the GSE17967 dataset, there were 291 upregulated and 658 downregulated HCC-specific mRNAs (Figure 1(b); Table S6). We initially identified 121 miRNAs from GSE63046 (Figure 1(c)) and 106 miRNAs from the GSE21362 data set (Figure $1(\mathrm{~d})$ ), respectively. The intersections of the two datasets of differentially expressed miRNAs consisted of $36(18.8 \%)$ miRNAs (5 upregulated and 31 downregulated, Figure 1(e); Table S7). These data provided a new clue to reveal the essential genes involved in the sequential progression from liver cirrhosis to HCC. 
SERHL, $P=0.000254$

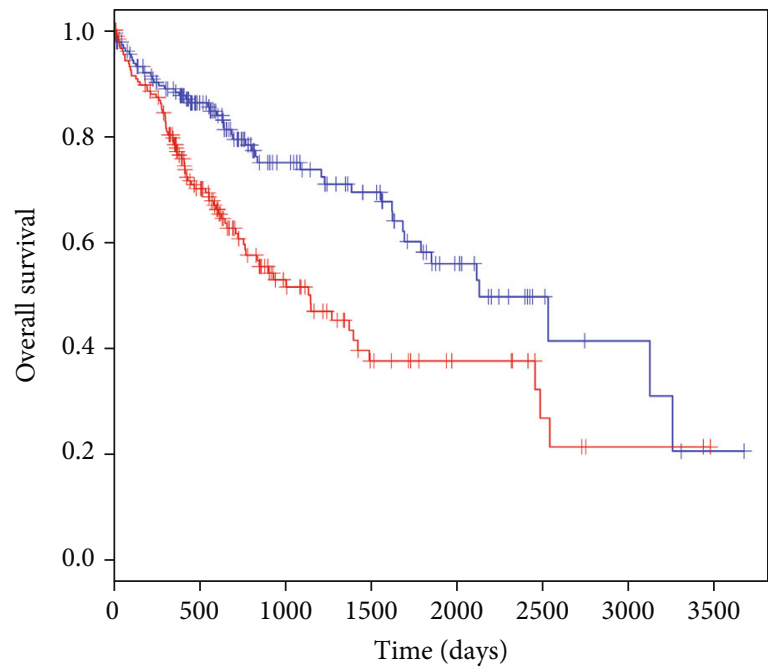

— High expression

— Low expression

(a)

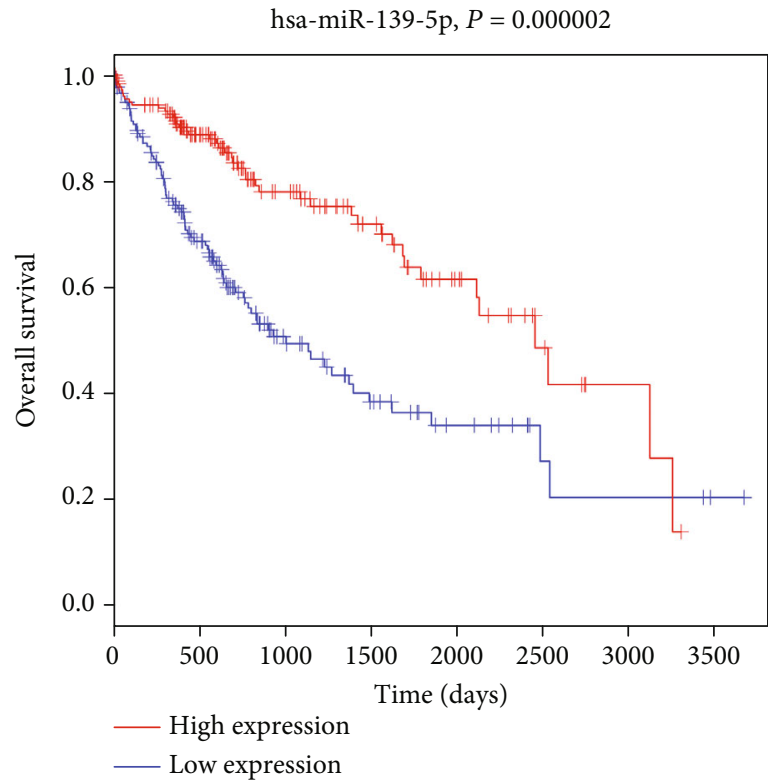

(c)
EGOT, $P=0.041800$

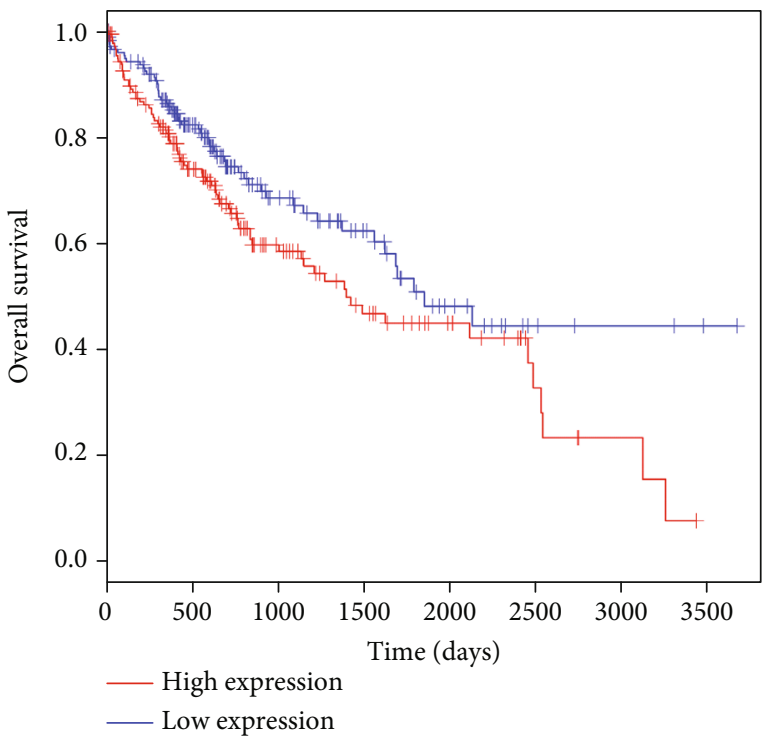

(b)

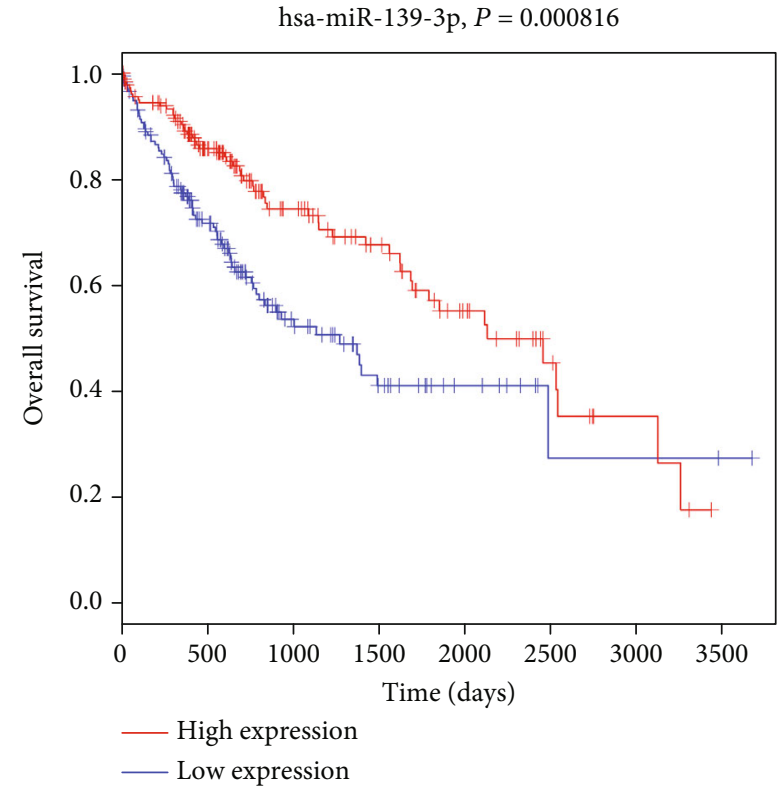

(d)

Figure 3: Continued. 


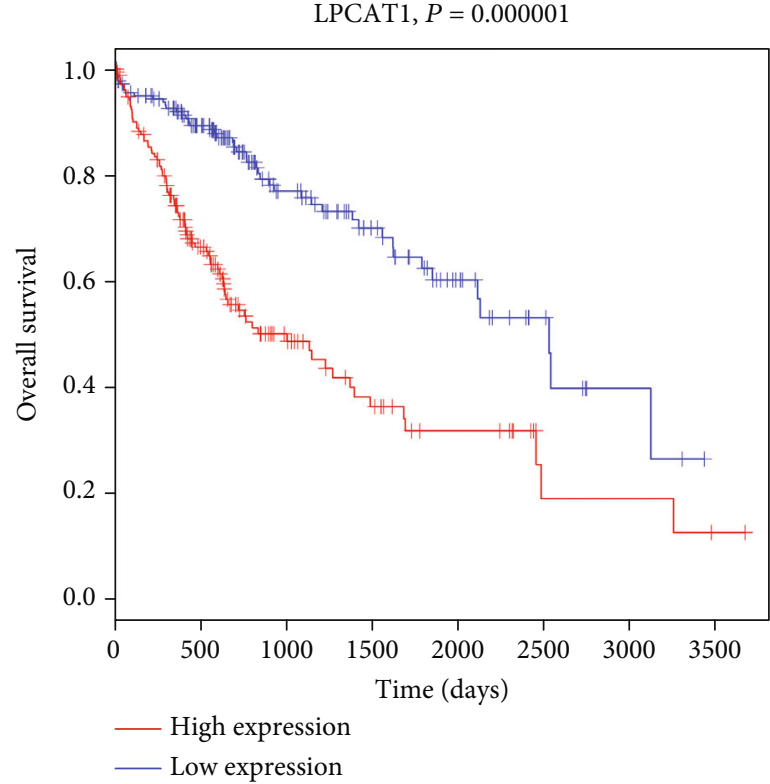

(e)

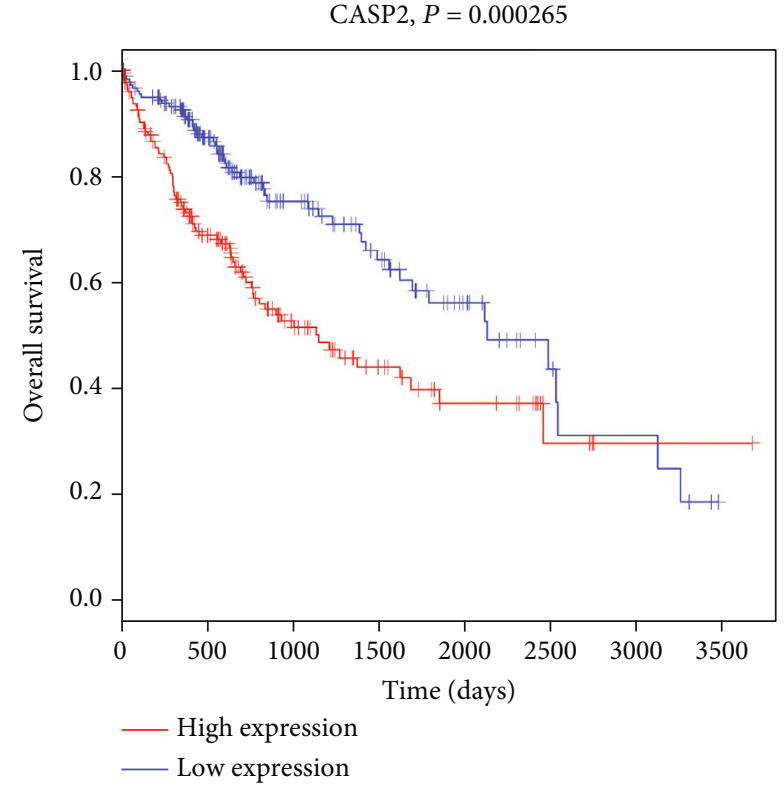

(f)

FIgURe 3: Prognosis prediction for differentially expressed ceRNAs. Kaplan-Meier survival curves for top two lncRNAs (a, b), miRNAs (c, d), and mRNAs $(e, f)$ associated with overall survival. Horizontal axis, overall survival time, days; vertical axis, survival function. Red represents high expression samples, and blue represents low expression samples. ceRNA: competing endogenous RNA; lncRNA: long noncoding RNA; miRNA: microRNA; mRNA: messenger RNA.

3.2. Construction of ceRNA Network. The lncRNA-mRNA coexpression network was built by differentially expressed lncRNAs and mRNAs to investigate the critical regulatory relations. A total of 16 lncRNAs (6 upregulated lncRNAs; 10 downregulated lncRNAs) and 219 mRNAs (96 upregulated mRNAs; 123 downregulated mRNAs) were enrolled in this network for further analysis. Next, based on miRanda and TargetScan analysis, the intersection differentially expressed miRNAs were used to predict the miRNAs negative targeted mRNAs, identifying 35 miRNAs negatively correlated to 360 mRNAs. Subsequently, we confirmed 36 miRNAs and the corresponding negative regulation to 69 lncRNAs. The lncRNA-miRNA-mRNA ceRNA coexpression network was built up based on the targeted interactions. We identified that 47 lncRNAs had regulatory effects on 35 intersection miRNAs (Table 2). Next, 35 miRNAs were interacting with 168 specific mRNAs (Table 3 ). Finally, the numbers of lncRNAs, miRNAs, and mRNAs were decreased to 47,35 , and 168 , respectively (Figure 2).

3.3. Prognosis Prediction for Differentially Expressed IncRNAs, miRNAs, and mRNAs. To identify the HCC-specific ceRNAs with prognostic characteristics, we enrolled 377 cirrhotic HCC patients from the TCGA-LIHC database, and the univariate Cox regression model was implemented based on the expression of these lncRNAs, miRNAs, and mRNAs, which illustrated the relationship between key ceRNAs and overall survival of HCC patients. Our results demonstrated that 2 lncRNAs, 4 miRNAs, and 40 mRNAs were significantly correlated to the overall survival in patients with cirrhotic HCC (Fig. S3). The expressions of top two lncRNAs (EGOT and SERHL), top two miRNAs (hsa-miR-139-5p and
hsa-miR-139-3p), and top two mRNAs (LPCAT1 and CASP2) were depicted in Figure $3(P<0.05)$. And KaplanMeier survival analyses revealed that these two lncRNAs, EGOT and SERHL, had negative correlation with overall survival in the progression of HCC. According to pathway analysis, we further demonstrated the expression pattern of EGOT and SERHL lncRNAs as well as their pair subnetworks (EGOT-miR-32-5p-XYLT2 axis and SERHL-miR1269a/miR-193b-3p-BCL2L1/SYK/ARNT/CHST3/LPCAT1 axis) (Fig. S4).

3.4. The Associations between Key IncRNAs and Clinical Characteristics. Multivariate Cox analysis was implemented to investigate the predictors of a clinical prognosis of cirrhotic HCC (Table S8). Due to less than 10 points of all VIF in the multivariate Cox model, there was no evidence of multicollinearity in independent variables. Compared with stage I, a later pathologic stage of stages III and IV was associated with a lower overall survival rate (HR 1.59, 95\% CI 1.05-2.43, and $P=0.029$ and HR 4.80, 95\% CI 1.4915.45 , and $P=0.009$, respectively), while there was no significant different in the pathologic stage of stage II (HR $0.93,95 \%$ CI $0.58-1.50, P=0.766)$. In patients with cirrhotic HCC, a higher alpha-fetoprotein (AFP) (HR 1.73, 95\% CI 1.18-2.55, $P=0.005)$ and a higher albumin (HR $2.45,95 \%$ CI $1.61-3.72, P<0.001)$ were related to a lower overall survival rate. In Cox hazard modeling for survival free from total mortality, the unadjusted HR for highexpressed EGOT group was 1.48 (95\% CI 1.05-2.09, $P=0.027$ ), which decreased to 1.35 (95\% CI 0.95-1.92, $P=0.091)$ in a multivariable model that included pathologic stage, AFP, and albumin (Table S3). The 

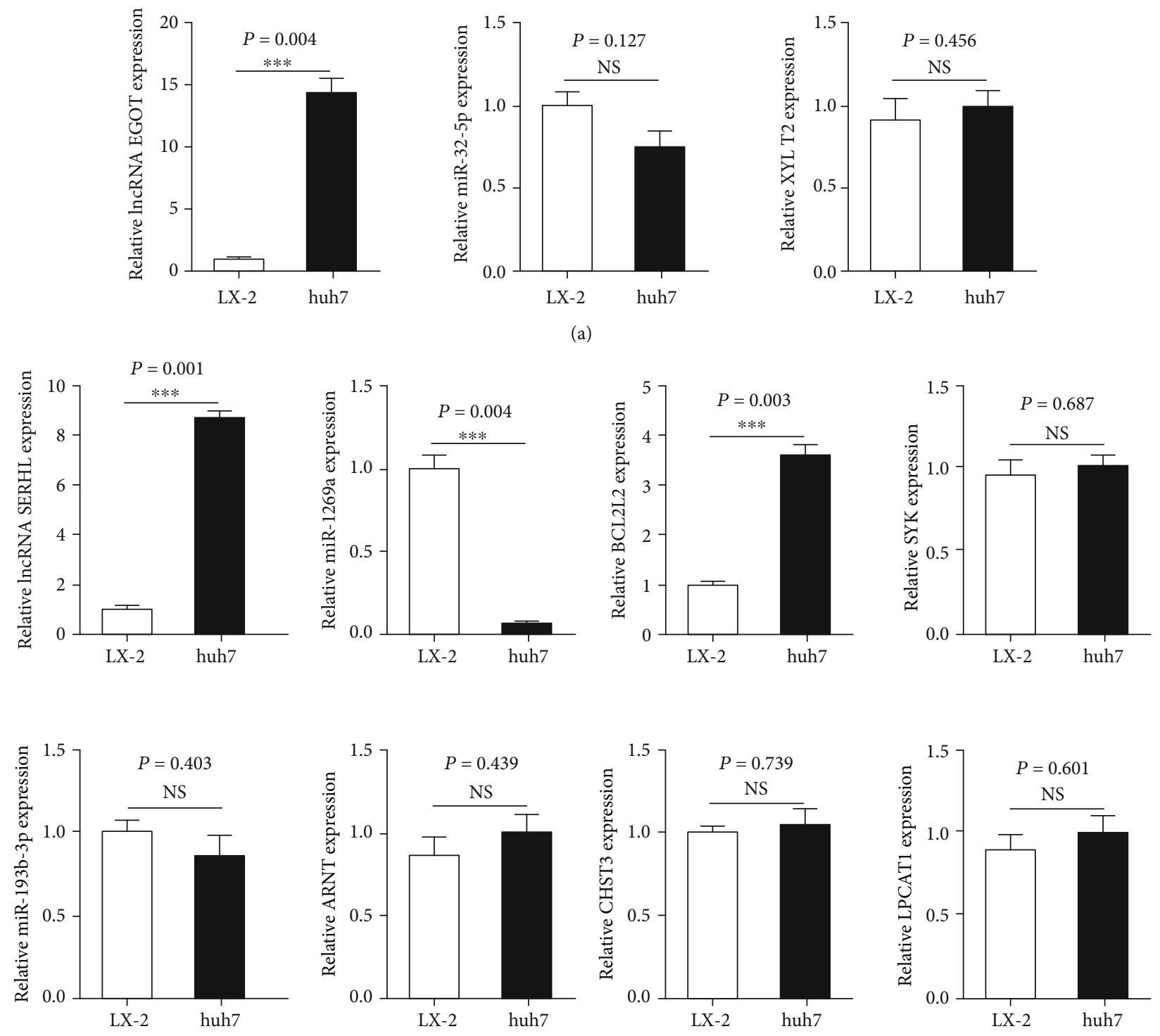

(b)

FIGURE 4: qRT-PCR validation of two key lncRNAs and their pair subnetworks in vitro studies. (a) Comparison of the expression levels of lncRNA EGOT-miR-32-5p-XYLT2 axis between Huh7 cells and LX-2 cells. (b) Comparison of the expression levels of lncRNA SERHLmiR-1269a/miR-193b-3p-BCL2L1/SYK/ARNT/CHST3/LPCAT1 axis between Huh7 cells and LX-2 cells. qRT-PCR: quantitative real-time polymerase chain reaction; lncRNA: long noncoding RNA.

unadjusted HR for high-expressed SERHL group was 1.97 (95\% CI $1.39-2.81, P<0.001$ ), which decreased to 1.74 (95\% CI 1.22-2.49, $P=0.002)$ in the adjusted multivariable model. A nomogram was then constructed by using AFP, serum albumin, pathologic stage, and lncRNA EGOT (Fig. S5A) or SERHL (Fig. S5B) to visualize the prediction model of 5-year survival probability.

3.5. $q R T-P C R$ Verification. IncRNA EGOT-miR-32-5pXYLT2 axis and lncRNA SERHL-miR-1269a/miR-193b-3pBCL2L1/SYK/ARNT/CHST3/LPCAT1 axis were selected to establish the validity and reliability of our results. The expressions of two key lncRNAs and their pair subnetworks were evaluated in Huh7 cells compared to LX-2 cells. As shown in Figure 4(a), IncRNA EGOT was significantly higher in Huh7 cells than in LX-2 cells $(P=0.004)$, suggesting that lncRNA EGOT was upregulated in human hepatoma cells compared with human hepatic fibrosis cells in vitro studies. However, the expressions of miR-32-5p and XYLT2 were not significantly different in Huh7 cells and LX-2 cells $(P=0.127$ and $P=0.456$, respectively). Similarly, lncRNA SERHL and BCL2L1 were significantly higher expressed ( $P=0.001$ and $P=0.003$, respectively), and miR-1269a was significantly lower expressed $(P=0.004)$ in Huh7 cells than in LX-2 cells (Figure 4(b)). However, no significant differences were found in the other subnetwork's expression, including miR-193b-3p, ARNT, CHST3, LPCAT1, and SYK (Figure 4(b)). 


\section{Discussion and Conclusions}

Our study demonstrated the differential expression profiling of lncRNAs, miRNAs, and mRNAs in cirrhotic HCC. Next, based on miRanda and TargetScan, we explored the coexpression regulatory relationships between lncRNA, miRNA, and mRNA. Then, we built a functional ceRNA network to provide a global view of HCC-associated differentially expressed RNAs with interregulated 47 lncRNAs, 35 miRNAs, and 168 mRNAs. We also uncovered that two differentially expressed lncRNAs (EGOT and SERHL), and their pair subnetworks could affect the prognosis of cirrhotic HCC and predict the overall survival of patients with HCC. This finding provides a novel insight into the initiation and development of cirrhotic HCC.

Emerging evidences indicate that the aberrant expression of lncRNAs, developing into a highly active research hotspot, plays significant roles in tumorigenesis and acts as novel molecular biomarkers for the diagnosis and prognosis of HCC. More recently, a growing body of research shows that the construction of the ceRNA network reveals its diagnostic and prognostic value in HCC by comprehensive analysis [14-17]. For example, lncRNA SSTR5-AS1, acting as a ceRNA, sponge mir-15b-5p to regulate carbonic anhydrase 2 (CA2) function in the progression of hepatitis B virusrelated hepatocellular carcinoma [18].

In the present study, we highlighted lncRNAs EGOT and SERHL might be two critical lncRNAs that act as diagnostic and prognostic biomarkers in the progression of liver cirrhosis to HCC. Previous work has demonstrated that lncRNA EGOT is involved in the progress of breast cancer [19], gastric cancer [20], head and neck squamous cell carcinomas [21], renal cell carcinoma [22], and hepatitis $C$ virus-induced liver tumors [23]. Moreover, higher expression of lncRNA SERHL is significantly correlated with patient's shorter overall survival in HCC, not involving in further research on the functional implications of SERHL [24]. Our results suggest that lncRNA EGOT-miR-32-5p-XYLT2 axis and lncRNA SERHL-miR-1269a/miR-193b-3p-BCL2L1/SY-

K/ARNT/CHST3/LPCAT1 axis could contribute to the regulatory mechanism underlying the pathogenetic process of HCC. IncRNA EGOT synergistically increased the expression of hsa-miR-32-5p and then decreased the expression of XYLT2, relating to significant regulation of key HCC-related signaling pathways such as glycosaminoglycan biosynthesis and metabolic pathways (Fig. S4). IncRNA SERHL synergistically upregulated the expression of hsa-miR-1269a and hsamiR-193b-3p and then downregulated the expression of BCL2L1, SYK, ARNT, CHST3, and LPCAT1, which are associated with the significant regulation of key HCC-related signaling pathways such as Ras signaling pathway, PI3K-Akt signaling pathway, NF-kappa B signaling pathway, and JAKSTAT signaling pathway (Fig. S4). Consistent with previous research, IncRNAs EGOT and SERHL are negatively associated with the overall survival of patients with cirrhotic HCC. These two lncRNAs may be exploited as potential effective biomarkers in the diagnosis and prognosis of HCC.

In conclusion, we identified the differential expression of cancer-specific lncRNAs, miRNAs, and mRNAs in the pro- gression of liver cirrhosis to HCC and constructed the ceRNA network to reveal their genetic interactions. The higher-expressed SERHL was associated with a higher risk of all-cause death. The expression of SERHL-miR-1269aBCL2L1 was significantly related to cirrhotic HCC in vitro studies. Importantly, as candidate biomarkers for HCC prognosis prediction, two potential unexplored lncRNAs EGOT and SERHL showed significantly negative associations with overall survival, and their pair subnetworks could provide a novel clue to future study into the underlying regulatory mechanism of cirrhotic HCC.

$\begin{array}{ll}\text { Abbreviations } \\ \text { HCC: } & \text { Hepatocellular carcinoma } \\ \text { lncRNAs: } & \text { Long noncoding RNAs } \\ \text { ceRNA: } & \text { Competing endogenous RNA } \\ \text { miRNA: } & \text { MicroRNA } \\ \text { mRNA: } & \text { Messenger RNA } \\ \text { TCGA: } & \text { The Cancer Genome Atlas } \\ \text { GEO: } & \text { Gene Expression Omnibus } \\ \text { FC: } & \text { Fold change } \\ \text { FBS: } & \text { Fetal bovine serum } \\ \text { SD: } & \text { Standard deviation } \\ \text { IQR: } & \text { Interquartile range } \\ \text { VIF: } & \text { Variance inflation factor } \\ \text { HR: } & \text { Hazard ratios } \\ \text { CI: } & \text { Confidence interval. }\end{array}$

\section{Data Availability}

The datasets of lncRNAs, miRNAs, and mRNAs have been contributed by GSE17967, GSE21362, GSE63046, and TCGA-LIHC. The data of the current study are available from the corresponding author on reasonable request.

\section{Conflicts of Interest}

There are no potential conflicts of interest.

\section{Authors' Contributions}

Yuli Zhang performed the experiments, analyzed the data, and wrote the manuscript. Dinggui Chen, Miaomiao Yang, Xianfeng Qian, and Chunmei Long performed the data processing and statistical analysis. Zhongwei Zheng designed the study, supervised the experiments, and wrote the manuscript.

\section{Acknowledgments}

The datasets of lncRNAs, miRNAs, and mRNAs have been contributed by GSE17967, GSE21362, GSE63046, and TCGA-LIHC. We thank all investigators for sharing these published data. This work was supported by the Changzhou Guidance Program of Applied Basic Research (CJ20209026 to Y.L.Z.). 


\section{Supplementary Materials}

The file of Supplementary Materials. (Supplementary Materials)

\section{References}

[1] C. Fitzmaurice, C. Allen, R. M. Barber et al., "Global, regional, and national cancer incidence, mortality, years of life lost, years lived with disability, and disability-adjusted life-years for 32 cancer groups, 1990 to 2015: a systematic analysis for the global burden of disease study," JAMA Oncology, vol. 3, no. 4, pp. 524-548, 2017.

[2] F. Bray, J. Ferlay, I. Soerjomataram, R. L. Siegel, L. A. Torre, and A. Jemal, "Global cancer statistics 2018: GLOBOCAN estimates of incidence and mortality worldwide for 36 cancers in 185 countries," CA: a Cancer Journal for Clinicians, vol. 68, no. 6, pp. 394-424, 2018.

[3] H. B. El-Serag and K. L. Rudolph, "Hepatocellular carcinoma: epidemiology and molecular carcinogenesis," Gastroenterology, vol. 132, no. 7, pp. 2557-2576, 2007.

[4] G. Fattovich, T. Stroffolini, I. Zagni, and F. Donato, "Hepatocellular carcinoma in cirrhosis: incidence and risk factors," Gastroenterology, vol. 127, no. 5, pp. S35-S50, 2004.

[5] X. Shi, M. Sun, H. Liu, Y. Yao, and Y. Song, "Long non-coding RNAs: a new frontier in the study of human diseases," Cancer Letters, vol. 339, no. 2, pp. 159-166, 2013.

[6] L. J. Lim, S. Y. S. Wong, F. Huang et al., "Roles and regulation of long noncoding RNAs in hepatocellular carcinoma," Cancer Research, vol. 79, no. 20, pp. 5131-5139, 2019.

[7] C. Li, J. Chen, K. Zhang, B. Feng, R. Wang, and L. Chen, "Progress and prospects of long noncoding RNAs (lncRNAs) in hepatocellular carcinoma," Cellular Physiology and Biochemistry, vol. 36, no. 2, pp. 423-434, 2015.

[8] L. Salmena, L. Poliseno, Y. Tay, L. Kats, and P. P. Pandolf, “A ceRNA hypothesis: the Rosetta Stone of a hidden RNA language?," Cell, vol. 146, no. 3, pp. 353-358, 2011.

[9] Y. Bai, J. Long, Z. Liu et al., "Comprehensive analysis of a ceRNA network reveals potential prognostic cytoplasmic lncRNAs involved in HCC progression," Journal of Cellular Physiology, vol. 234, no. 10, pp. 18837-18848, 2019.

[10] K. J. Archer, V. R. Mas, K. David, D. G. Maluf, K. Bornstein, and R. A. Fisher, "Identifying genes for establishing a multigenic test for hepatocellular carcinoma surveillance in hepatitis C virus-positive cirrhotic patients," Cancer Epidemiology Biomarkers \& Prevention, vol. 18, no. 11, pp. 2929-2932, 2009.

[11] F. Sato, E. Hatano, K. Kitamura et al., "MicroRNA profile predicts recurrence after resection in patients with hepatocellular carcinoma within the Milan Criteria," PLoS One, vol. 6, no. 1, p. e16435, 2011.

[12] A. Wojcicka, M. Swierniak, O. Kornasiewicz et al., "Next generation sequencing reveals microRNA isoforms in liver cirrhosis and hepatocellular carcinoma," The International Journal of Biochemistry \& Cell Biology, vol. 53, pp. 208-217, 2014.

[13] P. Shannon, A. Markiel, O. Ozier et al., "Cytoscape: a software environment for integrated models of biomolecular interaction networks," Genome Research, vol. 13, no. 11, pp. 24982504, 2003.

[14] J. Long, Y. Bai, X. Yang et al., "Construction and comprehensive analysis of a ceRNA network to reveal potential prognostic biomarkers for hepatocellular carcinoma," Cancer Cell International, vol. 19, no. 1, pp. 1-12, 2019.
[15] X. Liao, X. Wang, K. Huang et al., "Integrated analysis of competing endogenous RNA network revealing potential prognostic biomarkers of hepatocellular carcinoma," Journal of Cancer, vol. 10, no. 14, pp. 3267-3283, 2019.

[16] X.-L. Ming, Y.-L. Feng, D.-D. He et al., "Role of BCYRN1 in hepatocellular carcinoma pathogenesis by lncRNA-miRNAmRNA network analysis and its diagnostic and prognostic value," Epigenomics, vol. 11, no. 10, pp. 1209-1231, 2019.

[17] C. Yue, Y. Ren, H. Ge et al., "Comprehensive analysis of potential prognostic genes for the construction of a competing endogenous RNA regulatory network in hepatocellular carcinoma," Oncotargets and Therapy, vol. Volume 12, pp. 561$576,2019$.

[18] J. Xu, J. Zhang, F. Shan, J. Wen, and Y. Wang, "SSTR5-AS1 functions as a ceRNA to regulate CA2 by sponging miR-15b$5 \mathrm{p}$ for the development and prognosis of HBV-related hepatocellular carcinoma," Molecular Medicine Reports, vol. 20, no. 6, pp. 5021-5031, 2019.

[19] S. Xu, J. Zhang, S. Sui et al., "Downregulation of the long noncoding RNA EGOT correlates with malignant status and poor prognosis in breast cancer," Tumor Biology, vol. 36, no. 12, pp. 9807-9812, 2015.

[20] W. Peng, J. Wu, H. Fan, J. Lu, and J. Feng, "LncRNA EGOT promotes tumorigenesis via hedgehog pathway in gastric cancer," Pathology \& Oncology Research, vol. 25, no. 3, pp. 883887, 2019.

[21] T. Kolenda, M. Kopczyńska, K. Guglas et al., "EGOT lncRNA in head and neck squamous cell carcinomas," Polish Journal of Pathology, vol. 69, no. 4, pp. 356-365, 2018.

[22] L. Jin, J. Quan, X. Pan et al., "Identification of lncRNA EGOT as a tumor suppressor in renal cell carcinoma," Molecular Medicine Reports, vol. 16, no. 5, pp. 7072-7079, 2017.

[23] E. Carnero, M. Barriocanal, C. Prior et al., "Long noncoding RNA EGOT negatively affects the antiviral response and favors HCV replication," EMBO Reports, vol. 17, no. 7, pp. 1013$1028,2016$.

[24] Y. Ma, T. Luo, D. Dong, X. Wu, and Y. Wang, "Characterization of long non-coding RNAs to reveal potential prognostic biomarkers in hepatocellular carcinoma," Gene, vol. 663, pp. $148-156,2018$. 